\section{The H1N1 factor and inverse correlation between death rates due to COVID-19 and influenza-pneumonia}

\author{
Ajit Haridas ${ }^{1}$ and Gangan Prathap ${ }^{2, *}$ \\ ${ }^{1} 42$ Vrindavan Gardens B, Thiruvananthapuram 695 004, India \\ ${ }^{2}$ A. P. J. Abdul Kalam Technological University, \\ Thiruvananthapuram 695 016, India
}

In an earlier study, we had observed an inverse correlation between death rates due to COVID-19 and influenza, suggesting cross-immunity. We examine virus surveillance data further to identify the key subtype of influenza virus that seems to give such crossimmunity. This is identified as the H1N1 strain and we show that a country where this strain was recently dominant has much lower COVID-19 mortality rates. It is possible to argue that many countries which already have a high burden of influenza and pneumonia-related deaths, may have a substantial fraction of the population immune to the $S A R S-C o V-2$ virus and hence experience lower mortality rates at the peak typical of exponentially growing epidemics. This has implication on policies appropriate for managing the epidemic.

Keywords: COVID-19, death rates, H1N1 subtype, influenza, pneumonia.

IN a recent unrefereed study, Haridas and Prathap ${ }^{1}$ showed that there is a strong and discernible inverse correlation between COVID-19 death rates and those due to influenza in many countries. An important implication is that several countries, including tropical countries like India with an already high burden of influenza and pneumonia-related deaths, would be less affected by the SARS-CoV-2 virus. Another important takeaway is that, if COVID-19 death rates are indeed inversely related to those due to influenza-pneumonia, then there is the factor of 'cross-immunity' or 'cross-protection' to be considered, that prior infection with one virus affords protection against closely related ones. Unlike the COVID-19 pandemic, the $2009 \mathrm{H} 1 \mathrm{~N} 1$ pandemic affected younger adults. This has been attributed to cross-immunity from infection by the prior $1950 \mathrm{H} 1 \mathrm{~N} 1$ influenza strain ${ }^{2}$. Immunological cross-reactivity of dengue virus antibodies to Zika virus (ZIKV) has been reported ${ }^{3}$. Cross-protection between successive waves of the 1918 Spanish flu pandemic has been elucidated from epidemiological data ${ }^{4}$. Morales et $a{ }^{5} .^{5}$ carried out a modelling study to identify factors contributing to country-wise variation in mortality in case of the $2009 \mathrm{H} 1 \mathrm{~N} 1$ pandemic. They found that age structure contributed $40 \%$ of the variation. Mortality was prominently impacted by co-circulating influenza sub-

*For correspondence. (e-mail: gangan_prathap@hotmail.com) types $\mathrm{H} 3 \mathrm{~N} 2$ and influenza B. There was a protective effect to the 2009 pandemic in countries where H3N2 dominated in 2008. Interestingly, they found no association between pandemic mortality in 2009 and factors such as antiviral stockpiling, medical and economic factors (e.g. health expenditure), international air travel, previous circulation of seasonal $\mathrm{H} 1 \mathrm{~N} 1$, or pandemic timing in a country - the start and the peak ${ }^{5}$. In a major review of the lessons learnt from the 1918 influenza pandemic, Short et $a l{ }^{6}$ remark that pre-existing immunity is likely to contribute to a more accurate prediction of viral severity even before the influenza virus in question becomes established as a pandemic.

Skountzou et $a .^{2}$ studied cross-protection against the pandemic swine-origin $2009 \mathrm{H} 1 \mathrm{N1}$ influenza virus A/California/04/09 provided by H1N1 influenza viruses that circulated in the human population from 1930 to 2000. They showed that mice exposed to the 1947 virus, $\mathrm{A} / \mathrm{FM} / 1 / 47$, or the 1934 virus, $\mathrm{A} / \mathrm{PR} / 8 / 34$, survived a lethal challenge with the 2009 virus. Conversely, mice exposed to the $2009 \mathrm{H} 1 \mathrm{~N} 1$ virus were protected against a lethal challenge with mouse-adapted 1947 or 1934 H1N1 viruses. In addition, exposure to the $2009 \mathrm{H} 1 \mathrm{~N} 1$ virus induced broad cross-reactivity against H1N1 as well as H3N2 influenza viruses. Finally, they showed that vaccination with the older H1N1 viruses, particularly $\mathrm{A} / \mathrm{FM} / 1 / 47$, conferred protective immunity against the 2009 pandemic H1N1 virus. Their data offered an explanation for the decreased susceptibility of the elderly to the 2009 pandemic.

Priyamvada et al. $^{3}$ addressed the issue of crossreactivity between dengue virus (DENV) and ZIKV by testing sera and plasmablast-derived monoclonal antibodies from dengue patients against ZIKV. They showed that both acute and convalescent dengue sera potently bind and neutralize ZIKV. The cross-reactivity was evident at the monoclonal level. Their findings that pre-existing dengue antibodies may modulate immune responses to ZIKV infection are highly relevant as majority of regions experiencing Zika virus epidemics are endemic for dengue.

The statistical inverse correlations seen between COVID-19 deaths and H1N1 infections and deaths suggest that there is a factor of cross-protection or preexisting immunity involved, and this points the way to better interventions at the level of public health.

As done in our earlier study ${ }^{1}$, we obtained influenza and pneumonia-related death rates per 100,000 of population for a cohort of 147 countries from the portal https:// www.worldlifeexpectancy.com/cause-of-death/influenzapneumonia/by-country/

We obtained the count of deaths from COVID-19 as of 16 April 2020 for the same cohort of 147 countries from https://www.worldometers.info/coronavirus/. Influenza and pneumonia are a natural cause of death in old age in many countries, irrespective of the quality of health care, 
and it is reasonable to expect that the same is true with the new SARS-CoV-2.

Figure 1 shows the scatter plot of COVID-19 deaths per 100,000 of population versus the number of deaths per year per 100,000 of population caused by influenzapneumonia for which data are available. Some remarks about scale-dependent stratification and the skylineshoreline delineation ${ }^{7}$ may be in order here before we proceed further. In bibliometric and econometric studies, there is a huge skew in the data as well as order-ofmagnitude changes in scale and a significant stratification between well-defined limit boundaries ${ }^{7}$. The limits of the scatter can be identified as skyline and shoreline boundaries. A similar order-of-magnitude stratification appears in the present study and the scale-dependent stratification classification is used here ${ }^{7}$. In Figure 1, we have also marked the trend lines (that come from conventional regression analyses), and skylines (shown here as an ultimate line) and shorelines of the scale-dependent stratification. Tracking over the last six weeks (results not shown here) has shown that the skyline moves in an almost parallel manner reflecting the increase in COVID19 mortality with progress in time.

Given the observation of an inverse correlation between COVID-19 per capita deaths and influenza per capita mortality, and assuming that it implies cross-immunity, we examined the influence of the type of influenza virus co-circulating in the immediate 28 weeks (week 40, 2019 to week 16,2020 ) prior to the COVID-19 epidemic. The WHO Global Influenza Surveillance and Response System (GISRS) (www.who.int/flunet), provides data on weekly distribution of influenza virus types, classified

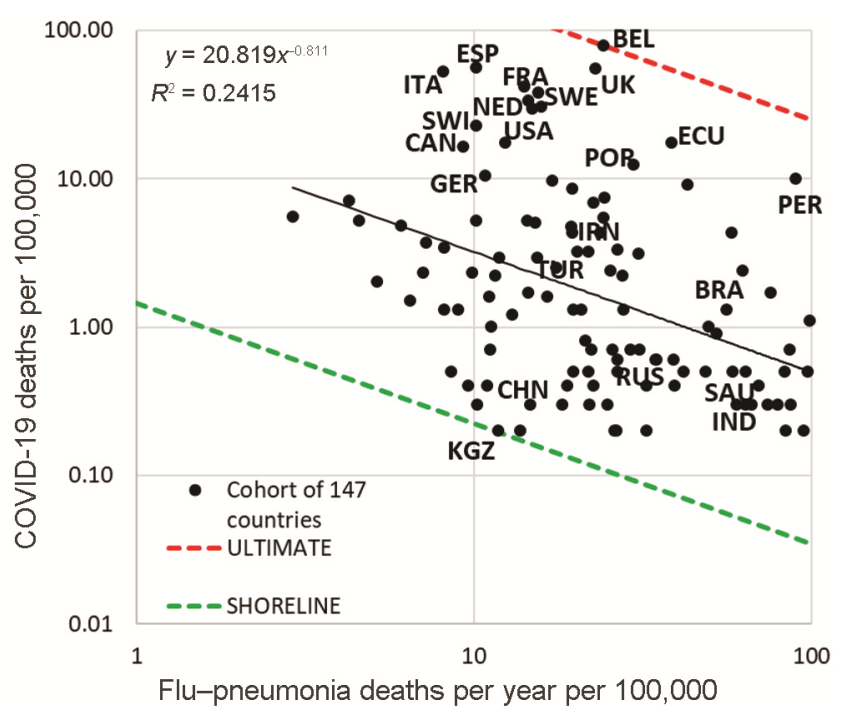

Figure 1. Scatter plot of COVID-19 deaths per 100,000 of population versus the number of deaths per year per 100,000 of population caused by Influenza-pneumonia for a cohort of 147 countries for which data are available. into A (H1), A (H1N1)pdm09, A (H3), A (H5), A (not subtyped), A (Total), B (Yamagata lineage), B (Victoria lineage), B (lineage not determined), B (Total). The data are available across a large number of countries. However, data on intensity of influenza (influenza cases per $100,000)$ in the immediate months prior to the COVID-19 pandemic are not available for most countries. In the absence of direct data, we cannot explicitly determine the correlation between virus subtype and immunity. We calculate a dominance number for $\mathrm{H} 1 \mathrm{~N} 1$ as follows

$$
D_{\mathrm{H} 1 \mathrm{~N} 1}=\frac{\sum_{40 / 2019}^{16 / 2020} N_{\mathrm{H} 1 \mathrm{~N} 1}}{\sum_{40 / 2019}^{16 / 2020}\left(N-N_{\mathrm{notA}}\right)},
$$

where $N$ is the total number of samples from the week tested, $N_{\mathrm{H} 1 \mathrm{~N} 1}$ the number of $\mathrm{H} 1 \mathrm{~N} 1$ positive samples, and $N_{\text {notA }}$ is the number of samples positive for type A, but not subtyped.

When the subtyping is done, this gives us a measure of the extent to which H1N1 dominates among the many possible types that co-circulate.

Table 1 shows deaths due to COVID-19 as of 16 April 2020 from https://www.worldometers.info/coronavirus/ and influenza-pneumonia deaths per year per 100,000 of population from https://www.worldlifeexpectancy.com/ cause-of-death/influenzapneumonia/by-country/. H1N1 dominance values are from www.who.int/flunet. Based on the H1N1 values we have clubbed the countries into three groups as high, mid and low H1N1 dominance countries. Reliable data were available only for 20 countries and hence only those are reported. In the more general case where the H1N1 factor was not explored, as reported in Haridas and Prathap ${ }^{1}$, we performed the analysis for 147 countries for which COVID-19 and influenza-pneumonia data were available, and the same inverse relationship held true.

Figure 2 shows a scatter plot of the flu or pneumonia deaths per 100,000 of population against the SARS-CoV2 deaths on 16 April 2020 for the 20 countries where we could get meaningfully documented subtype data for flu infection. As in Haridas and Prathap ${ }^{1}$, it was found that countries with higher influenza/pneumonia death rates had much lower death rates due to SARS-CoV-2. Part of the scatter in Figure 2 is explained by the difference in the timing - start date - of the epidemic. Nevertheless, countries like the Philippines, Singapore, Malaysia, Japan and Korea have been exposed for durations longer or comparable to Italy and Spain, but showed much lower mortality rates due to COVID-19. The clustering of Japan and Korea in the green category of having recent H1N1 co-circulation, with Singapore, Thailand and Indonesia is evident. Iran had a recent $\mathrm{H} 1 \mathrm{~N} 1$ co-circulation and death rates from COVID-19 were less than the UK, with 
Table 1. Deaths due to COVID-19 as of 16 April 2020 from https:/www.worldometers.info/coronavirus/ and due to flu-pneumonia from https://www.worldlifeexpectancy.com/cause-of-death/influenzapneumonia/by-country/. H1N1 dominance values are estimated from

\begin{tabular}{|c|c|c|c|c|c|}
\hline Country & Code & $\begin{array}{c}\mathrm{H} 1 \mathrm{~N} 1 \\
\text { dominance }\end{array}$ & $\begin{array}{l}\text { Influenza-pneumonia deaths } \\
\text { per year per } 100,000 \text { of } \\
\text { population }\end{array}$ & $\begin{array}{l}\text { COVID-19 deaths as of } \\
16 \text { April } 2020\end{array}$ & $\begin{array}{l}\text { COVID-19 deaths per } \\
100,000 \text { of population as } \\
\text { of } 16 \text { April } 2020\end{array}$ \\
\hline Norway & NOR & 0.09 & 19.62 & 150 & 2.8 \\
\hline USA & USA & 0.14 & 14.91 & 28,554 & 8.6 \\
\hline Great Britain & GBR & 0.15 & 23.01 & 12,868 & 19.0 \\
\hline Ireland & IRE & 0.11 & 15.91 & 444 & 9.0 \\
\hline France & FRA & 0.27 & 14.1 & 17,167 & 26.3 \\
\hline Portugal & POR & 0.18 & 29.81 & 599 & 5.9 \\
\hline Italy & ITA & 0.26 & 8.15 & 21,645 & 35.8 \\
\hline Spain & ESP & 0.48 & 10.21 & 18,812 & 40.2 \\
\hline Philippines & PHL & 0.25 & 115.6 & 362 & 0.3 \\
\hline Peru & PER & 0.31 & 90.3 & 254 & 0.8 \\
\hline Brazil & BRA & 0.35 & 42.96 & 1,760 & 0.8 \\
\hline Germany & GER & 0.42 & 10.86 & 3,804 & 4.5 \\
\hline Malaysia & MAL & 0.58 & 74.61 & 84 & 0.3 \\
\hline Thailand & THA & 0.66 & 69.87 & 46 & 0.1 \\
\hline Indonesia & IDN & 0.70 & 26.72 & 498 & 0.2 \\
\hline Korea & KOR & 0.71 & 19.84 & 229 & 0.4 \\
\hline Iran & IRN & 0.72 & 19.67 & 4,777 & 5.7 \\
\hline Singapore & SIN & 0.80 & 70.09 & 10 & 0.2 \\
\hline Ecuador & $\mathrm{ECU}$ & 0.83 & 38.7 & 388 & 2.2 \\
\hline Japan & JPN & 0.88 & 34.54 & 178 & 0.1 \\
\hline
\end{tabular}

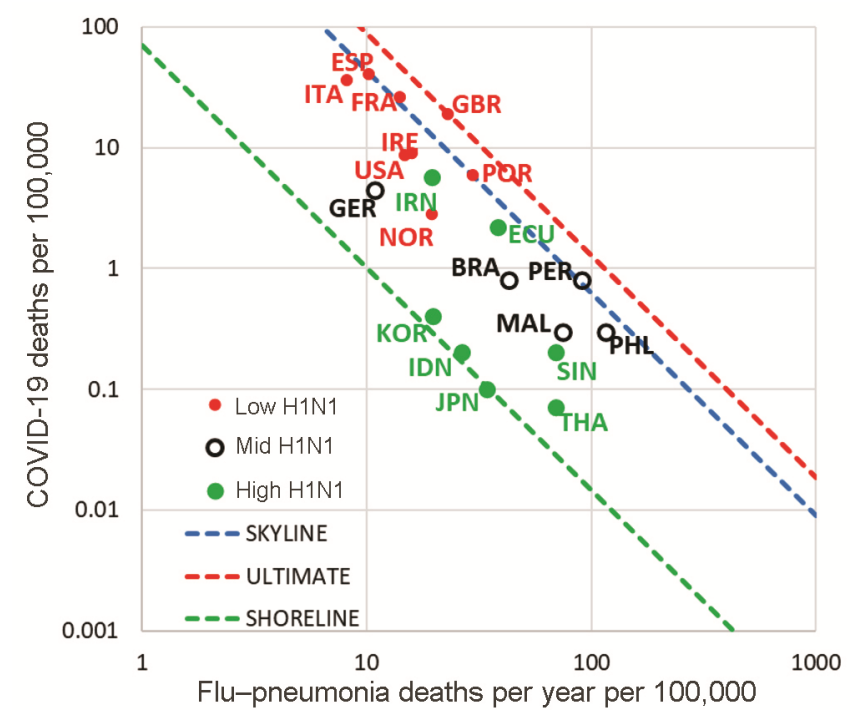

Figure 2. Scatter plot of COVID-19 deaths per 100,000 of population versus the number of deaths per year per 100,000 of population caused by influenza-pneumonia.

comparable flu death rates. Germany is an outlier as regards COVID-19 mortality, and it is the only Western European country with substantial recent H1N1 as the dominant co-circulating virus. The higher than expected COVID-19 mortality in the Philippines matches the lesser dominance of H1N1.

In order to see the H1N1 effect separately, we show in Figure 3 the scatter plot of COVID-19 deaths per 100,000 of population versus $\mathrm{H} 1 \mathrm{~N} 1$ dominance. The clustering is clearly brought here. The green 'low H1N1' points lie on the right end of the $X$-axis indicating that higher H1N1 dominance suggests greater immunity and therefore fewer deaths.

In the absence of data on the extent of H1N1 infection, including mild infections not captured in hospitalization data, we have made a proxy estimate of the seasonal H1N1 infection rate per 100,000 of population as given by $D_{\mathrm{H} 1 \mathrm{~N} 1} F$, where $\mathrm{F}$ is the average annual influenza death rate per 100,000 of population. Figure 4 shows the scatter plot of COVID-19 deaths per 100,000 of population versus the proxy for H1N1 flu-pneumonia deaths per year per 100,000 of population.

The power trendline (using the feature available in Excel) in Figure 4 clearly shows the inverse relationship for all country data points taken together. As before we can also draw a limiting skyline (shown as ultimate) that connects four countries at nearly the same phase of the epidemic by visual inspection using the same exponent as that of the trendline. The distance from the country datapoint to this skyline represents how far the epidemic has progressed in attaining the expected 'annual' death rate.

While the distance to the skyline would depend on when the first infection took place, the group of countries, viz. Korea, Japan, the Philippines, Malaysia, Singapore and Thailand, are among those that had early first reported cases of COVID-19. Yet, they are relatively distant from the skyline. This implies that the epidemic has been very slow in these countries. It is well known from epidemic models that the positive term in the equation for 
rate of growth of infected population, commonly referred to as time-dependent reproduction number $R$, is proportional to the fraction of population who are susceptible. Pre-existing immunity reduces the fraction of population who are susceptible and reduces the rate of infection. The distance from the skyline in Figure 4 reveals the effect, and therefore, we may infer that these countries have sufficient immunity and will observe a steady rate of COVID-19 cases. Epidemiological models show that when substantial immunity exists in the population, an epidemic takes a trajectory of rise in mortality followed by steady plateau or slow decrease. The result implies that Government policies designed for dampening peak morbidity are not appropriate for countries with high

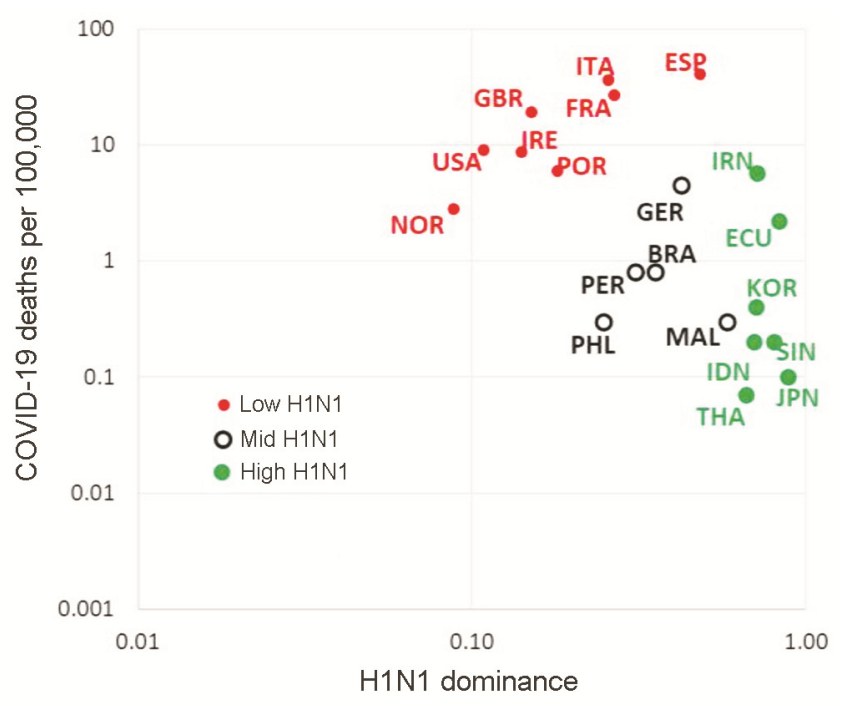

Figure 3. Scatter plot of COVID-19 deaths per 100,000 of population versus $\mathrm{H} 1 \mathrm{~N} 1$ dominance.

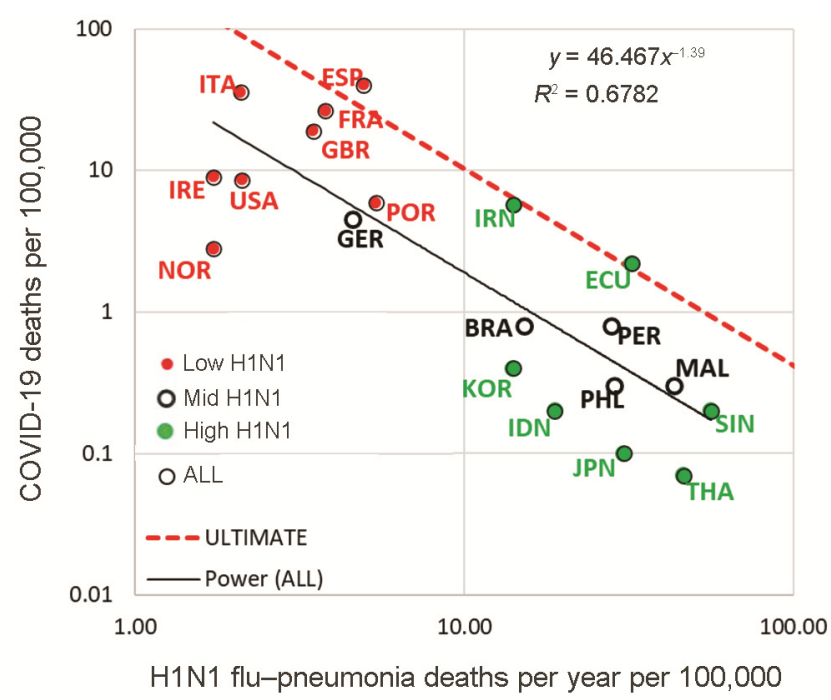

Figure 4. Scatter plot of COVID-19 deaths per 100,000 of population versus the proxy for H1N1 influenza-pneumonia deaths per year per 100,000 of population. incidence of influenza and, in particular, H1N1, as they would experience a steady infection and death rate. When a substantial fraction of the population has pre-existing immunity through exposures that confer cross-immunity, we would expect slow spread of the disease, similar to endemic infection patterns. Correspondingly, the benefits of restriction on economic activities are limited.

Antibodies with specific affinity to SARS-CoV-2 develop by a process of selection of increasing affinity when challenged by a viral antigen. The presence of antibodies with a weak affinity to the new virus gives a head start to the immune response. In epidemiological language, this translates to lower morbidity and lower mortality. While the genetic origins of SARS-CoV-2 and $\mathrm{H} 1 \mathrm{~N} 1$ are different, it is possible that the 'antigenic distance' may be close. What Singapore and Malaysia influenza data show (the rates being higher than those for India, which has far inferior health-care facilities) is that influenza as the cause of death is almost independent of the quality of health care - availability and access to critical care. Influenza is kept under check by the immune system. Basically, the body must protect itself against influenza, not doctors or ventilators.

An important caveat has been brought to our notice by an anonymous reviewer. It is that in complex systems it is common that a statistical pattern seen has many alternative interpretations. The present study establishes H1N1 factor as one that influences the inverse relationship in mortality between COVID-19, and influenza-pneumonia. However, this need not be the only one controlling such inverse relationship. Ethnicity could be a factor influencing the negative correlations, e.g. Caucasoid and East Asian can be differently clustered in all graphs. However, this could be taken up as a future line of study. We expect that the history of exposure to infections would be much more significant than ethnicity in determining the immunity of the population. Separately, we have studied the role of population age structure, but no negative trends were found. We are currently studying the under 5-yrs mortality rate; this also has a significant inverse relationship with COVID-19 mortality rates and the results will be communicated separately. We have also studied factors such as urbanization, availability of critical-care facilities (e.g. hospital beds per thousand of population and physicians per thousand of population), population age structure, but failed to find any significant inverse trends for COVID-19 mortality.

Using data available in the public domain (https://www. worldlifeexpectancy.com/cause-of-death/influenza-pneumonia/by-country/, https://www.worldlifeexpectancy. com/cause-of-death/influenza-pneumonia/by-country/ and https://www.who.int/flunet) we have shown that so far COVID-19 death rates are inversely related to those due to influenza and pneumonia, and that the H1N1 strain of the virus plays a key role in this. If suggests that there is the factor cross-immunity or cross-protection to be 
considered, that prior infection with one virus affords protection against closely related ones.

The main conclusions from this study can be summarized as follows: The variation in COVID-19 per capita death rates in various countries appears to be of natural origin, namely immunity status of the population arising from its history of exposure to infections. This factor accounts for orders of magnitude differences in per capita deaths due to the COVID-19 pandemic, and needs to be considered in formulation of policies for the management of the pandemic. Policies that restrict economic activity to avoid overloading of health infrastructure are appropriate only where the expected rates of serious infection and death are substantial. It would obviously not deliver benefits commensurate with costs in countries where the per capita deaths are low, as a result of pre-existing immunity.

Declaration: The authors declare no competing interests.

1. Haridas, A. and Prathap, G., Are COVID-19 rates inversely proportional to the death rates by flu and influenza?, 2020; https://www. researchgate.net/publication/340262817; doi:10.13140/RG.2.2.16795. 21285.

2. Skountzou, I. et al., Immunity to pre-1950 H1N1 influenza viruses confers cross protection against the pandemic swine-origin 2009 A (H1N1) influenza virus. J. Immunol., 2010, 185, 1642-1649.

3. Priyamvada, L. et al., Human antibody responses after dengue virus infection are highly cross-reactive to Zika virus. Proc. Natl. Acad. Sci. USA, 2016, 113, 7852-7857.

4. Barry, J. M., Viboud, C. and Simonsen, L., Cross-protection between successive waves of the 1918-1919 influenza pandemic: epidemiological evidence from US Army camps and from Britain. $J$. Infect. Dis., 2008, 198, 1427-1434.

5. Morales, K. F., Paget, J. and Spreeuwenberg, P., Possible explanations for why some countries were harder hit by the pandemic influenza virus in 2009 - a global mortality impact modeling study. BMC Infect. Dis., 2017, 17, 642-644.

6. Short, K. R., Kedzierska, K. and van de Sandt, C. E., Back to the future: lessons learned from the 1918 influenza pandemic. Front. Cell. Infect. Microbiol., 2018, 8, 343; doi:10.3389/fcimb.2018. 00343.

7. Prathap, G., Scale-dependent stratification: a skyline-shoreline scatter plot. Scientometrics, 2019, 119(2), 1269-1273.

Received 25 April 2020; revised accepted 17 June 2020

doi: $10.18520 / \mathrm{cs} / \mathrm{v} 119 / \mathrm{i} 3 / 535-539$

\section{Space-based observations on the impact of COVID-19-induced lockdown on aerosols over India}

\author{
Yogesh Kant*, Debashis Mitra and \\ Prakash Chauhan \\ Indian Institute of Remote Sensing, \\ Indian Space Research Organisation, Department of Space, \\ Dehradun 248 001, India
}

The lockdown period in India due to COVID-19 came into effect from 25 March 2020 onwards. The present study analyses the changes and trends in aerosol optical depth (AOD) levels during the last few months and particularly during lockdown period. MODIS observations showed an average reduction of $20-37 \%$ in aerosol loading during the lockdown period (25 March-3 May 2020), compared to 2017-2019 across India. A clear-cut and drastic reduction in AOD (which includes both PM2.5 and PM10) was observed at many places across India, in particular over North India (46\%), eastern Indo-Gangetic Plains (42\%) and peninsular India $(30 \%)$. Maximum decrease in AOD was seen during 25 March to 5 April 2020 with values as low as $0.18-0.22$, which are $72-87 \%$ lower than the February-March 2020 level. AOD then slightly increased in the third week of April due to stubble burning and a few cloudy days, and then fell subsequently. Ground measurements on air pollutants at selected Indian cities revealed reduction of $40-50 \%$ in surface concentration prior to the lockdown period. Thus, study shows a clear-cut improvement in air quality leading to significant improvement in visibility and more blue skies.

Keywords: Aerosol optical depth, air quality, coronavirus, lockdown.

THE coronavirus (COVID-19) has infected over 4.8 million people worldwide causing more than 0.318 million deaths and spreading to all corners of the Earth (as on 19 May 2020) ${ }^{1}$. Depending on the level of infection and impact of COVID-19, each country has been adopting different levels of intervention, including national and international road, rail and air travel, complete or partial lockdown of people's movement, industrial and economic activity, markets and social activities. Since the reporting of the deadly coronavirus from Wuhan, China on 31 December 2019 and its subsequent spread to various parts of the world, the World Health Organisation (WHO) declared it as pandemic on 12 March 2020. The first case of COVID-19 in India was reported on 30 January 2020, which rose to 110 cases by 15 March and $500+$ active cases by 24 March 2020 (ref. 2). From 25 March 2020 onwards the Government of India (GoI) announced a

*For correspondence. (e-mail: ykanty@gmail.com) 